\title{
Asymptotically optimal tree-packings in regular graphs
}

\author{
Alexander Kelmans * \\ Rutgers University, New Brunswick, New Jersey and \\ University of Puerto Rico, San Juan, Puerto Rico \\ kelmans@rutcor.rutgers.edu \\ Dhruv Mubayi ${ }^{\dagger}$ \\ School of Mathematics, Georgia Institute of Technology, Atlanta, GA 30332-0160, USA \\ Microsoft Research, One Microsoft Way, Redmond, WA 98052-6399 \\ mubayi@microsoft.com \\ Benny Sudakov $\ddagger$ \\ Department of Mathematics, Princeton University, Princeton, NJ 08544, USA \\ and Institute for Advanced Study, Princeton, NJ 08540, USA \\ bsudakov@math.princeton.edu \\ Submitted: February 15, 2001; Accepted: November 21, 2001. \\ AMS Subject Classifications: 05B40, 05C05, 05C35, 05C70, 05D15 \\ Keywords: Packing trees, matchings in hypergraphs
}

\begin{abstract}
Let $T$ be a tree with $t$ vertices. Clearly, an $n$ vertex graph contains at most $n / t$ vertex disjoint trees isomorphic to $T$. In this paper we show that for every $\epsilon>0$, there exists a $D(\epsilon, t)>0$ such that, if $d>D(\epsilon, t)$ and $G$ is a simple $d$-regular graph on $n$ vertices, then $G$ contains at least $(1-\epsilon) n / t$ vertex disjoint trees isomorphic to $T$.
\end{abstract}

\section{Introduction}

We consider simple undirected graphs. Given a graph $G$ and a family $\mathcal{F}$ of graphs, an $\mathcal{F}$-packing of $G$ is a subgraph of $G$ each of whose components is isomorphic to a member of $\mathcal{F}$. The $\mathcal{F}$-packing problem is to find an $\mathcal{F}$-packing of the maximum number of vertices. There are various results on the $\mathcal{F}$-packing problem (see e.g. $[3,9,10,11,12,13,14,15]$ ).

\footnotetext{
${ }^{*}$ Research supported in part by the National Science Foundation under DIMACS grant CCR 91-19999.

${ }^{\dagger}$ Research supported in part by the National Science Foundation under grant DMS-9970325.

${ }_{\ddagger}^{\ddagger}$ Research supported in part by NSF grants DMS-0106589, CCR-9987845 and by the State of New Jersey.
} 
When $\mathcal{F}$ consists of a single graph $F$, we abuse notation by writing $F$-packing. The very special case of the $F$-packing problem when $F=K_{2}$, a single edge, is simply that of finding a maximum matching. This problem is well-studied, and can be solved in polynomial time (see, for example, [15]). However, if $F$ is a connected graph with at least three vertices then the $F$-packing problem is known to be NP-hard [13]. The $F$-packing problem remains NP-hard even for 3-regular graphs if $F$ is a path with at least 3 vertices [11].

There are various directions for studying this generally intractable problem. One possible direction is to try to obtain bounds on the size of the maximum $F$-packing of various families of graphs, as well as the corresponding polynomial approximation algorithms. The following is an example of such a result. It concerns the $P_{3}$-packing problem for 3-regular graphs, where $P_{3}$ is the 3 -vertex path.

Theorem 1.1. [12] Suppose that $G$ is a 3-regular graph. Then $G$ contains at least $v(G) / 4$ vertex disjoint 3-vertex paths that can be found in polynomial time (and so for 3-regular graphs there is a polynomial approximation algorithm that guarantees at least a 3/4optimal solution for the $P_{3}$-packing problem).

Another direction is to consider some special classes of graphs in hope to find a polynomial time algorithm for the corresponding $F$-packing problem. Here is an example of such a result.

Theorem 1.2. [9] Suppose that $G$ is a claw-free graph (i.e. $G$ contains no induced subgraph isomorphic to $\left.K_{1,3}\right)$. Suppose also that $G$ is connected and has at most two endblocks (in particular, 2-connected). Then the maximum number of disjoint 3-vertex paths in $G$ is equal to $\lfloor v(G) / 3\rfloor$ vertex disjoint 3-vertex paths. Moreover there is a polynomial time algorithm for finding an optimal $P_{3}$-packing in $G$.

An asymptotic approach provides another direction for studying this $N P$-hard problem. There is a series of interesting asymptotic packing results on sufficiently dense graphs. They have beed iniciated by the following deep theorem of Hajnal and Szemerédi.

Theorem 1.3. [8] If $G$ has $n$ vertices and minimum degree at least $(1-1 / r) n$, then $G$ contains $\lfloor n / r\rfloor$ vertex-disjoint copies of $K_{r}$.

Theorem 1.3 has been generalized by Alon and Yuster for graphs other than $K_{r}$.

Theorem 1.4. [2] For every $\gamma>0$ and for every positive integer $h$, there exists an $n_{0}=n_{0}(\gamma, h)$ such that for every graph $H$ with $h$ vertices and for every $n>n_{0}$, any graph $G$ with hn vertices and with minimum degree $\delta(G) \geq(1-1 / \chi(H)+\gamma)$ hn contains $n$ vertex-disjoint copies of $H$.

In this paper we consider an asymptotic version of the $F$-packing problem, where $F$ is a tree. Our main result is the following.

Theorem 1.5. Let $T$ be a tree on $t$ vertices and let $\epsilon>0$. Suppose that $G$ is a d-regular graph on $n$ vertices and $d \geq \frac{128 t^{3}}{\epsilon^{2}} \ln \left(\frac{128 t^{3}}{\epsilon^{2}}\right)$. Then $G$ contains at least $(1-\epsilon) n / t$ vertex disjoint copies of $T$. 
Both Theorem 1.3 and Theorem 1.4 require $G$ to have $\Omega\left(n^{2}\right)$ edges. Theorem 1.5 differs from these results in that our graphs are not required to be dense. Indeed, $d$ above is only a function of $\epsilon$ and the size of the tree and does not depend on $n$. Consequently, Theorem 1.5 cannot possibly be extended to graphs other than trees, since the Turán number of a cycle of length $2 t$ is known to be at least $\Omega\left(n^{(2 t+1) / 2 t}\right)$ [4], and there exist essentially regular graphs with about this many edges that contain no copy of $C_{2 t}$.

In this paper, we present two approaches for obtaining tree-packing results for regular graphs. First, in Section 2 we give a short proof of an asymptotic version of Theorem 1.5. This proof relies on powerful hypergraph packing results of Frankl and Rödl [7] and Pippenger and Spencer [17]. Next, in Section 3 we present a proof of Theorem 1.5, based on a probabilistic approach. It uses another powerful result called the Lovász Local Lemma (see e.g., [1]). In addition, it provides an explicit dependence of the degree on $t$ and $\epsilon$. Section 4 contains some concluding remarks and an open question.

\section{$2 \quad T$-packings from matchings in hypergraphs}

In this section we present the proof of the following asymptotic version of Theorem 1.5 .

Theorem 2.1. Let $T$ be a tree on $t$ vertices. Let $G_{n}$ be a $d_{n}$-regular graph on $n$ vertices. Suppose that $d_{n} \rightarrow \infty$ when $n \rightarrow \infty$. Then $G_{n}$ contains at least $(1-o(1)) n / t$ (and, obviously, at most $n / t$ ) disjoint trees isomorphic to $T$.

The proof of this theorem is based on a hypergraph packing result of Pippenger and Spencer [17]. The main idea behind this proof came from a result of Rödl [18] that solved an old packing conjecture of Erdős and Hanani [5]. Rödl's idea, now known as his "nibble", was used by Frankl-Rödl [7] to prove that under certain regularity and local density conditions, a hypergraph has a large matching. Pippenger and Spencer used probabilistic methods to extend and generalize the result in [7].

First we introduce some notions about hypergraphs. All hypergraphs we consider are allowed to have multiple edges. Given a hypergraph $\mathcal{H}=(V, E)$, the degree $d(v)$ of a vertex $v \in V$ is the number of edges containing $v$. For vertices $v, w$, the $\operatorname{codegree} \operatorname{cod}(v, w)$ of $v$ and $w$ is the number of edges containing both $v$ and $w$. Let

$$
\Delta(\mathcal{H})=\max _{v \in V} d(v), \quad \delta(\mathcal{H})=\min _{v \in V} d(v), \quad C(G)=\max _{u, v \in V, u \neq v} \operatorname{cod}(u, v) .
$$

A matching in $\mathcal{H}$ is a set of pairwise disjoint edges of $\mathcal{H}$. Let $\mu(\mathcal{H})$ be the size of the largest matching in $\mathcal{H}$. A matching $M$ is perfect if every vertex of $\mathcal{H}$ is in exactly one edge of $M$. A hypergraph $\mathcal{H}$ is $t$-uniform if each of its edges consists of exactly $t$ elements.

Theorem 2.2. [17] For every $t \geq 2$ and $\varepsilon>0$, there exist $\varepsilon^{\prime}>0$ and $n_{0}$ such that if $\mathcal{H}$ is a t-uniform hypergraph on $n(\mathcal{H}) \geq n_{0}$ vertices with $\delta(\mathcal{H}) \geq\left(1-\varepsilon^{\prime}\right) \Delta(\mathcal{H})$, and $C(\mathcal{H}) \leq \varepsilon^{\prime} \Delta(\mathcal{H})$, then

$$
\mu(\mathcal{H}) \geq(1-\varepsilon) n / t
$$


We rephrase Theorem 2.2 in more convenient asymptotic notation.

Theorem 2.1'. Let $\mathcal{H}_{1}, \mathcal{H}_{2}, \ldots$ be sequence of t-uniform hypergraphs, with $\left|V\left(\mathcal{H}_{k}\right)\right| \rightarrow \infty$. If $\delta\left(\mathcal{H}_{k}\right) \sim \Delta\left(\mathcal{H}_{k}\right)$, and $C\left(\mathcal{H}_{k}\right)=o\left(\Delta\left(\mathcal{H}_{k}\right)\right)$, then $\mu\left(\mathcal{H}_{k}\right) \sim\left|V\left(\mathcal{H}_{k}\right)\right| / t$.

The above result says that under certain regularity and local density conditions on $\mathcal{H}$, one can find an almost perfect matching $M$ in $\mathcal{H}$, i.e., the number of vertices in no edge of $M$ is negligible. In fact, [17] proves something much stronger, namely that one can decompose almost all the edges of $\mathcal{H}$ into almost perfect matchings, but we need only the weaker statement.

Next we show how Theorem 2.2 can be applied to provide asymptotically optimal tree-packings of regular graphs. For convenience, we omit the subscript $k$ and the use of integer parts in what follows. Our goal is to produce a large $T$-packing in $G$. By a copy of $T$ we mean a subgraph isomorphic to $T$.

Given $u, v \in V(G)$ let $c(v)$ and $c(u, v)$ denote the number of copies of $T$ in $G$ containing $v$ and $\{u, v\}$, respectively (note that different copies may have the same vertex set). The following lemma provides necessary estimates for the numbers $c(v)$ and $c(u, v)$.

Lemma 2.3. Let $T$ be a tree with $t$ vertices. Suppose that $G$ is a d-regular graph on $n$ vertices. Then

$(c 1) c(v)=(1+o(1)) c_{T} d^{t-1}(d \rightarrow \infty)$ for every $v \in V(G)$, where $c_{T}$ depends only on $T$ and does not depend on the choice of $v$, and

$(c 2) c(a, b)=O\left(d^{t-2}\right)$ for every pair $a, b \in V(G), a \neq b$.

Proof. We first estimate $c(v)$. Let us consider the rooted tree $R$ obtained from $T$ by specifying a vertex $r$ of $T$ as a root. Let $c_{r}(v)$ denote the number of copies of $R$ in $G$ in which the vertex $v \in V(G)$ is chosen to be the root $r$.

It is easy to see that $c(v)=\sum\left\{c_{r}(v) / g: r \in V(T)\right\}=(1+o(1))(t / g) d^{t-1}$, where $g$ is the size of the automorphism group of $T$. Therefore it suffices to show that $c_{r}(v)=$ $(1+o(1)) d^{t-1}$ for all $r \in V(T)$ and $v \in V(G)$.

Let $x_{1}$ be a leaf of $R$ distinct from $r, R_{1}=R-x_{1}$, and $y_{1}$ be the vertex in $R_{1}$ adjacent to $x_{1}$. If $\left(x_{i}, y_{i}, R_{i}\right)$ is already defined, let $x_{i+1}$ be a leaf of $R_{i}$ distinct from $r$, $R_{i+1}=R_{i}-x_{i+1}$, and $y_{i+1}$ be the vertex in $R_{i+1}$ adjacent to $x_{i+1}$. Clearly $r=y_{t-1}=R_{t-1}$. Now we estimate $c_{r}(v)$ as follows. There is only one way to allocate $r$ in $G$, namely, to allocate $r$ in $v$. Since $v$ is of degree $d$ in $G$ and $G$ is simple, there are $d$ ways to allocate $x_{t-1}$ in $G$. Suppose that $R_{i}, 1 \leq i<t-1$, is already allocated in $G$, and $y_{i}$ is allocated in a vertex $v_{i}$ in $G$. Since $v_{i}$ is of degree $d$ in $G$ and $G$ is simple, there are at most $d$ and at least $d-t+i$ ways to allocate $x_{i}$ in $G$. Therefore

$$
(d-t)^{t-1}<c_{r}(v)<d^{t-1}
$$

Since $d \rightarrow \infty$, we have: $c_{r}(v)=(1+o(1)) d^{t-1}$ for all $r \in V(T)$ and $v \in V(G)$.

Now we will estimate $c(a, b)$, the number of copies of $T$ in $G$ containing both $a$ and $b$ where $a \neq b$. For $x, y \in V(T)$, let $c_{x, y}(a, b)$ denote the number of copies of $T$ containing 
$a, b$, with $a$ playing the role of $x$ and $b$ playing the role of $y$. Clearly

$$
c(a, b) \leq\left(\begin{array}{l}
t \\
2
\end{array}\right) \max _{x, y \in V(T)} c_{x, y}(a, b)
$$

because $a, b$ play the role of some pair $x, y$ in each copy of $T$ containing them. Hence it suffices to show that $c_{x, y}(a, b) \leq d^{t-2}$.

Split $T$ in two nontrivial trees $X$ and $Y$ where $X$ is rooted at $x$ and $Y$ is rooted at $y$, $V(X) \cap V(Y)=\emptyset$, and $V(X) \cup V(Y)=V(T)$. This can be done by deleting any edge from the unique path between $x$ and $y$. By $(*)$, there are at most $d^{|V(X)|-1}$ copies of $X$ in $G$ with $a$ playing the role of $x$, and at most $d^{|V(Y)|-1}$ copies of $Y$ in $G$ with $b$ playing the role of $y$. Thus $c_{x, y}(a, b) \leq d^{|V(X)|-1} d^{|V(Y)|-1}=d^{t-2}$.

Proof of Theorem 2.1 Given $G$, we must find a $T$-packing of size at least $(1-o(1)) n / t$. From $G$ construct the hypergraph $\mathcal{H}=(V, E)$ with $V=V(G)$ and $E$ consisting of vertex sets of copies of $T$ in $G$ (note that $\mathcal{H}$ can have multiple edges). Then claim $(c 1)$ of Lemma 2.3 implies $\delta(\mathcal{H})=\Delta(\mathcal{H}) \sim c_{T} d^{t-1}$, and claim $(c 2)$ of Lemma 2.3 implies $C(\mathcal{H})=O\left(d^{t-2}\right)=o\left(d^{t-1}\right)=o(\Delta(\mathcal{H}))$. Hence, by Theorem 2.2, $\mu(\mathcal{H}) \sim|V(H)| / t=n / t$. This clearly yields a $T$-packing in $G$ of the required size.

\section{T-packings from the Lovász Local Lemma}

This section contains a proof of Theorem 1.5 based on a probabilistic approach and the so called Lovász Local Lemma. We use the following symmetric version of the Lovász Local Lemma.

Theorem 3.1. [1] Let $A_{1}, \ldots, A_{n}$ be events in a probability space. Suppose that each event $A_{i}$ is mutually independent of a set of all the other events $A_{j}$ but at most $d$, and that $\operatorname{Prob}\left[A_{i}\right] \leq p$ for all $i$. If ep $(d+1) \leq 1$, then $\operatorname{Prob}\left[\wedge \overline{A_{i}}\right]>0$.

Here we make no attempt to optimize our absolute constants. First we need the following lemma. Given a partition $V_{1}, \ldots, V_{t}$ of the vertex set of a graph $G$, let $d_{i}(v)$ denote the number of neighbors of a vertex $v$ of $G$ in $V_{i}$.

Lemma 3.2. Let $t$ be an integer and let $G$ be a d-regular graph satisfying $d \geq 4 t^{3}$. Then there exists a partition of $V(G)$ into $t$ subsets $V_{1}, \ldots, V_{t}$ such that

$$
\frac{d}{t}-4 \sqrt{\frac{d}{t} \ln d} \leq d_{i}(v) \leq \frac{d}{t}+4 \sqrt{\frac{d}{t} \ln d}
$$

for every $v \in V$ and $1 \leq i \leq t$.

Proof. Partition the set of vertices $V$ into $t$ subsets $V_{1}, V_{2}, \ldots, V_{t}$ by choosing for each vertex randomly and independently an index $i$ in $\{1, \ldots, t\}$ and placing it into $V_{i}$. For $v \in V(G)$ and $1 \leq i \leq t$, let $A_{i, v}$ denote the event that $d_{i}(v)$ is either greater than 
$\frac{d}{t}+4 \sqrt{\frac{d}{t} \ln d}$ or less than $\frac{d}{t}-4 \sqrt{\frac{d}{t} \ln d}$. Observe that if none of the events $A_{i, v}$ holds, then our partition satisfies the assertion of the lemma. Hence it suffices to show that with positive probability no event $A_{i, v}$ occurs. We prove this by applying Theorem 3.1.

Since the number of neighbors of any vertex $v$ in $V_{i}, i=1,2, \ldots, t$, is a binomially distributed random variable with parameters $d$ and $1 / t$, it follows by the standard Chernoff's-type estimates for Binomial distributions (cf. , e.g., [16], Theorem 2.3) that for every $v \in V$

$$
\operatorname{Pr}\left(\left|d_{i}(v)-\frac{d}{t}\right|>a \frac{d}{t}\right) \leq 2 e^{-\frac{a^{2}(d / t)}{2(1+a / 3)}} .
$$

By substituting $a$ to be $4 \sqrt{(t / d) \ln d}$, we obtain that the probability of the event $A_{i, v}$ is at most $2 e^{-4 \ln d}=2 d^{-4}$. Clearly each event $A_{i, v}$ is independent of all but at most $t d(d-1)$ others, as it is independent of all events $A_{j, u}$ corresponding to vertices $u$ whose distance from $v$ is larger than 2 . Since $e \cdot 2 d^{-4} \cdot(t d(d-1)+1)<e \cdot 2 d^{-4} \cdot t d^{2}<1$, we conclude, by Theorem 3.1, that with positive probability no event $A_{i, v}$ holds. This completes the proof of the lemma.

Next we prove the following tree-packing result for nearly-regular, $t$-partite graphs, which is interesting in its own right.

Theorem 3.3. Let $T$ be a fixed tree with the vertex set $u_{1}, \ldots, u_{t}$ and let $H$ be a $t$-partite graph with parts $V_{1}, \ldots, V_{t}$ such that $\left|V_{1}\right|=h$ and for every vertex $v \in V(H)$ and every $1 \leq i \leq t$ the number $d_{i}(v)$ of neighbors of $v$ in $V_{i}$ satisfies $(1-\delta) k \leq d_{i}(v) \leq(1+\delta) k$ for some $k>0$ and $0 \leq \delta<1$. Then $H$ contains $(1-2(t-1) \delta) h$ vertex disjoint copies of $T$ with the property that $V_{i}$ contains the vertex of each copy corresponding to $u_{i}, 1 \leq i \leq t$.

Proof. We use induction on $t$. For $t=1$ the assertion is trivially true. Therefore let $t \geq 2$. Without loss of generality, we can assume that $u_{t}$ is a leaf adjacent to the vertex $u_{t-1}$. Let $T^{\prime}=T-u_{t}$ and $H^{\prime}=H-V_{t}$. Then by the induction hypothesis, we can find at least $(1-2(t-2) \delta) h$ vertex disjoint copies of $T^{\prime}$ in $H^{\prime}$ such that in all these copies the vertices, corresponding to $u_{t-1}$, belong to $V_{t-1}$. Denote the set of these vertices by $S$. Consider all the edges between $S$ and $V_{t}$. In the resulting bipartite graph $B$ each vertex is of degree at most $(1+\delta) k$. Therefore the edges of $B$ can be covered by $(1+\delta) k$ disjoint matchings. In addition, note that each vertex from $S$ has degree at least $(1-\delta) k$. Since the number of edges in $B$ is at least $(1-\delta) k|S|$, we conclude that $B$ contains a matching of size at least

$$
\frac{(1-\delta) k|S|}{(1+\delta) k}=\frac{1-\delta}{1+\delta}|S| \geq(1-2 \delta)|S| .
$$

By adding the edges of this matching to the appropriate copies of $T^{\prime}$, we obtain at least $(1-2 \delta)|S|=(1-2 \delta)(1-2(t-2) \delta) h \geq(1-2(t-1) \delta) h$ vertex disjoint copies of $T$. This completes the proof of the statement.

Having finished all necessary preparations, we are now ready to complete the proof of Theorem 1.5.

Proof of Theorem 1.5. Let $G$ be a $d$-regular graph on $n$ vertices with $d \geq \frac{128 t^{3}}{\epsilon^{2}} \ln \left(\frac{128 t^{3}}{\epsilon^{2}}\right)$ and let $T$ be a tree with $t$ vertices. By Lemma 3.2, we can partition vertices of $G$ into 
$t$ parts $V_{1}, \ldots, V_{t}$ such that $\left|V_{1}\right| \geq n / t$ (pick $V_{1}$ to be the largest part) and for every vertex the number of its neighbors in $V_{i}, 1 \leq i \leq t$, is bounded by $(1 \pm \delta) d / t$, where $\delta=4 \sqrt{(t / d) \ln d} \leq \epsilon / 2 t$. Thus by Theorem 3.3, $G$ contains at least $(1-2(t-1) \delta)\left|V_{1}\right| \geq$ $(1-\epsilon) n / t$ vertex disjoint copies of $T$.

\section{Concluding remarks}

- The regularity requirement in Theorem 1.5 cannot be weakened to a minimum degree requirement. To see this, let $G_{d}$ be the complete bipartite graph with parts $X, Y$ of sizes $d$ and $d^{2}$, respectively. The minimum degree of $G_{d}$ is $d \rightarrow \infty$, but clearly the largest $T$-packing has size at most $d=o\left(\left|V\left(G_{d}\right)\right|\right)$. On the other hand, it is easy to see that the proof of Theorem 1.5 remains valid for nearly-regular graphs. More precisely one can show the following.

Proposition 4.1. Let $T$ be a tree on $t$ vertices. For all $t$ and $\epsilon>0$, there exist two positive numbers $\gamma=\gamma(t, \epsilon)$ and $D(t, \epsilon)$ such that the following holds: if $d>D(t, \epsilon)$ and $G$ is a graph on $n$ vertices with $(1-\gamma) d \leq \delta(G) \leq \Delta(G) \leq(1+\gamma) d$, then $G$ contains $(1-\epsilon) n / t$ vertex disjoint copies of $T$.

It is also easy to see that the above results can be extended to $d$-regular multigraphs provided all multiplicities are bounded.

- The dependency of the degree of the graph on both $t$ and $\epsilon$ is needed in the statement of Theorem 1.5. To see this, let $G$ be a regular graph consisting of $\lceil\epsilon n / t\rceil$ disjoint cliques of size $k$, where $k=\Theta(t / \epsilon)$ is an integer such that $k \equiv t-1(\bmod t)$. Clearly any packing of $G$ by a tree on $t$ vertices misses at least $t-1$ vertices in each clique. Therefore altogether it will miss at least $(t-1)(\epsilon n / t)=\Omega(\epsilon|V(G)|)$ vertices. This shows that in the statement of Theorem 1.5 the degree of the graph should be at least $\Omega(t / \epsilon)$. Thus there is a big gap between the upper and lower bounds and this leads to the following

Question. What is the correct dependency of the degree of the graph $G$ on $t$ and $\epsilon$ to guarantee $(1-\epsilon) n / t$ vertex disjoint copies of $T$ in $G$ ?

Acknowledgments. The first author thanks Michael Krivelevich for very useful remarks. The second author thanks Brendan Nagle for very helpful discussions and for pointing out some relevant references.

\section{References}

[1] N. Alon, J. Spencer, The Probabilistic Method, Wiley, New York, 1992.

[2] N. Alon, R. Yuster, H-factors in dense graphs, J. Combin. Theory Ser. B 66 (1996), no. 2, 269-282. 
[3] G. Cornuéjols and D. Hartvigsen, An extension of matching theory, J. Combin. Theory B 40 (1986) 285-296.

[4] P. Erdős, Graph theory and probability, Canadian J. Math. 11, 34-38.

[5] P. Erdős, H. Hanani, On a limit theorem in combinatorial analysis, Publ. Math. Debrecen 10 (1963), 10-13.

[6] P. Erdős, H. Sachs, Reguläre graphen gegenbener Taillenweite mit minimaler Knotenzahl, Wiss. Z. Uni. Halle (Math. Nat.) 12 (1963), 251-257.

[7] P. Frankl, V. Rödl, Near Perfect Coverings in Graphs and Hypergraphs, Europ. J. Combin. 6 (1985), 317-326.

[8] A. Hajnal, E. Szemerédi, Proof of a conjecture of P. Erdős, in:Combinatorial theory and its applications, II (Proc. Colloq., Balatonfüred, 1969), pp. 601-623. North-Holland, Amsterdam, 1970.

[9] A. Kaneko, A. Kelmans, and T. Nisimura, On packing 3-vertex paths in a graph, J. Graph Theory 36 (2001) 175-197.

[10] A. Kelmans, Optimal packing of induced stars in a graph, Discrete Mathematics, 173, (1997) 97-127.

[11] A. Kelmans, Packing $P_{k}$ in a cubic graph is NP-hard if $k \geq 3$, in print.

[12] A. Kelmans and D. Mubayi, How many disjoint 2-edge paths must a cubic graph have ?, submitted (see also DIMACS Research Report 2000-23, Rutgers University).

[13] D. G. Kirkpatrick, P. Hell, On the complexity of general graph factor problems, SIAM J. Comput. 12, (1983) 601-609.

[14] M. Loebl and S. Poljak, Efficient subgraph packing, J. Combin. Theory B 59 (1993) $106-121$.

[15] L. Lovasz, M. Plummer, Matching Theory, North-Holland, Amsterdam, 1986.

[16] C. McDiarmid, Concentration, in : Probabilistic Methods for Algorithmic Discrete Mathematics, pp. 195-248, Springer, Berlin, 1998.

[17] N. Pippenger, J. Spencer, Asymptotic behavior of the chromatic index for hypergraphs, J. Combin. Theory Ser. A 51 (1989), 24-42.

[18] V. Rödl, On a Packing and Covering Problem, Europ. J. Combin. 5 (1985), 69-78. 